\title{
Aprovechamiento del recurso hídrico en Costa Rica: el caso de los acueductos municipales
}

\author{
Use of water resource in Costa Rica: the case of municipal \\ aqueducts
}

\section{Orlando Josué Hernández Cruz}

\author{
Universidad de Costa Rica \\ San José, Costa Rica \\ orlando.hernandezcruz@ucr.ac.cr \\ Marie Laura Fernández Ramírez \\ Consultora independiente \\ San José, Costa Rica \\ mariefdz11@gmail.com
}

\begin{abstract}
RESUMEN. El propósito de este artículo es dar a conocer los resultados sobre el aprovechamiento del recurso hídrico por parte de los acueductos municipales en Costa Rica. Estos resultados son parte de una investigación que se realizó como seminario de graduación para la Escuela de Administración Pública. Aunque se reconoce la importancia de siempre tener presente el concepto de la gestión integral del recurso hídrico, en esta oportunidad se presentan solo los resultados sobre el aprovechamiento en función de la cobertura y cantidad, la calidad y el costo, y la cultura hídrica.
\end{abstract}

De los resultados principales, se resalta una cobertura limitada para estos acueductos municipales, la falta de mejoras en la medición para determinar el aprovechamiento real del recurso hídrico y, aunque se cuenta con buenos estándares de calidad, hace falta mejorar las tarifas y cobros para dar sostenibilidad a los sistemas de acueductos. En cuanto a cultura hídrica, se tienen resultados interesantes donde se denotan avances importantes tanto por parte de los acueductos municipales, así como en relación con el invo-

Este es un artículo de acceso abierto publicado bajo los términos de la Licencia Creative Commons 4.0 Internacional Atribución-NoComercial-SinDerivadas. 
lucramiento de la ciudadanía.

En conclusiones, faltan detalles por afinar en los acueductos municipales en cuanto al modelo de gestión, sin embargo, se debe tomar con mucha seriedad realizar inversiones en infraestructura, redefinición de la manera con la que se calculan y/o recaudan las tarifas para poder dar sostenibilidad, además de adquirir instrumentos de medición que permitan dar cuenta del aprovechamiento real que se tiene de este recurso.

PALABRAS CLAVE. Recurso hídrico, aprovechamiento, gestión pública, acueductos municipales, Costa Rica

ABSTRACT. The purpose of this article is to present the results on the use of water resources by municipal aqueducts in Costa Rica. These results are part of an investigation that was carried out as a seminar for the School of Public Administration. Although it is recognized the importance of always bearing in mind the concept of the integral management of water resources, in this opportunity only the results on the use of water according to its factors of coverage and quantity, quality and cost, and water culture are presented.

The main results encompass the limited coverage for these municipal aqueducts, the lack of improvement of the water meters to determine the real use of the hydro resource, and, although they count with good quality standards, it is necessary to improve the rates and charges to provide sustainability to the aqueduct systems. Regarding water culture, there are interesting results showing important advances for the municipal aqueducts, as well as in relation to citizen involvement.

In conclusion, there are many details to improve in the municipal aqueducts regarding the management model, yet, investments in infrastructure must be taken very seriously, redefining the way in which rates are calculated and / or collected in order to provide sustainability. Besides, it is necessary to invest in new measuring instruments that will provide real data acquisition and trustful information about the water use.

KEYWORDS. Water resource, use of water, public management, municipal aqueducts, Costa Rica

\section{INTRODUCCIÓN}

El presente artículo de investigación se desarrolla a partir de los resultados presentados en la memoria del Seminario de Graduación titulado: Análisis de la gestión local del recurso hídrico en los acueductos municipales: el caso de Costa Rica, de las sustentantes Stephanie Agüero Hurtado, Daniela Aragón Hernández, y, Marie Laura Fer- 
nández Ramírez en el año 2018. El Seminario de Graduación fue dirigido por el Dr. Orlando Josué Hernández Cruz.

Este trabajo se realiza en un contexto actual donde se debate si el recurso hídrico debe ser visto como un bien económico, como un bien público, o, como ambos, y, aún se discuten las implicaciones que tiene cada enfoque. Se realiza un análisis a diecinueve municipalidades que tienen a su cargo acueductos municipales. En el estudio original se trabajan los ejes relacionados con la administración, el aprovechamiento y la conservación del recurso hídrico. No obstante, el presente artículo se centra en los resultados obtenidos en relación con el aprovechamiento del recurso hídrico por parte de estas municipalidades.

Se parte del supuesto que señala Núñez (2011) sobre la importancia de tomar conciencia, que sin importar lo que se decida, el recurso hídrico es un recurso natural, vital para el desarrollo de la vida, de modo que su gestión debe garantizar ante todo la sostenibilidad de este.

Por lo tanto, se sustenta el trabajo de la investigación en lo establecido en la Ley No. 7554, Ley Orgánica del Ambiente, donde se indica que el agua es de dominio público, su conservación y uso sostenible son de interés social. Por lo cual, la Administración Pública debe intervenir en su modelo de gestión e ir adaptando cada elemento que compone el mismo en relación con el comportamiento de este recurso natural.

Además, se consideran y concurren con posiciones de una diversidad de autores y autoras relacionados a la gestión integral del recurso hídrico, entre ellos y ellas, se encuentran: Ramos y León (2016); Morales, García y Carreón (2014); Morales (2010); Echeverría y Cantillo (2013); Carvajal, García y Jiménez (2007).

Para Ramos y León (2016), la gestión integral del recurso hídrico toma relevancia en las últimas décadas para las personas encargadas de gestionarlo, esto debido a que, para la fecha, la demanda del recurso natural está aumentando y sus fuentes están disminuyendo, a pesar de que el $70 \%$ del planeta está cubierto de agua solo el 2,5 $\%$ de esta potable. Por su parte, Morales, García y Carreón (2014), establecen que la problemática en la gestión del agua se debe a la disminución paulatina de disponibilidad del recurso, debido a que la explotación del recurso es superior a su recarga natural. Por lo cual, estas personas indican que se debe implementar una adminis- 
tración consensuada para atacar la escasez y abastecimiento.

Desde otra perspectiva, Morales (2010), indica que en Costa Rica hay abundancia de recurso hídrico, sin embargo, sostiene que no se encuentra bien distribuido ni geográfica ni estacionalmente. Se presume que la ausencia de ordenamiento territorial, la deforestación y los cambios en el uso del agua, así como el deterioro de las cuencas hidrográficas producto de la falta de planificación y la contaminación, son algunos de los factores que amenazan el recurso hídrico en el país. Además, el autor manifiesta que Costa Rica tiene complicaciones respecto a la

dispersión de autoridad, descoordinación interinstitucional, serios rezagos en inversión pública, falta de un programa de monitoreo sistemático que permita conocer la cantidad y la calidad del recurso, la legislación dispersa, obsoleta y la poca voluntad política para invertir en infraestructura y recursos humanos. (p.2)

Es por lo tanto necesario procurar una adecuada gestión del agua ya que, en coherencia con Carvajal, García y Jiménez (2007), la vulnerabilidad del recurso hídrico aumenta en sistemas mal administrados, que están bajo tensión o cuya ordenación es deficiente e insostenible. Así que, partiendo de lo anterior, se hace aún más relevante lo establecido en la Constitución Política de Costa Rica (1949) en su artículo 50:

El Estado procurará el mayor bienestar a todos los habitantes del país, organizando y estimulando la producción y el más adecuado reparto de la riqueza. Toda persona tiene derecho a un ambiente sano y ecológicamente equilibrado...El Estado garantizará, defenderá y preservará ese derecho.

A lo cual se adiciona la reforma constitucional a este artículo mediante el artículo $1^{\circ}$ de la ley $\mathrm{N}^{\circ} 9849$ del 5 de junio del 2020, "Reconocer y garantizar el derecho humano de acceso al agua":

Toda persona tiene el derecho humano, básico e irrenunciable de acceso al agua potable, como bien esencial para la vida. El agua es un bien de la nación, indispensable para proteger tal derecho humano. Su uso, protección, sostenibilidad, conservación y explotación se regirá por lo que establezca la ley que se creará para estos efectos y tendrá prioridad el abastecimiento de agua potable para consumo 
de las personas y las poblaciones.

El aprovechamiento del recurso hídrico se torna esencial debido a que se presume que el uso correcto del recurso permitirá mejorar en los indicadores necesarios para darle sostenibilidad en el tiempo a los acueductos municipales que fueron objeto de la investigación.

En Costa Rica coexisten diversos actores que operan el recurso hídrico, entre ellos: el Instituto Costarricense de Acueductos y Alcantarillados (AyA), Comités de Acueductos Rurales o Asociaciones Administradoras de Acueductos, Municipalidades, Empresa de Servicios Públicos de Heredia, y otros operadores privados.

Una cantidad importante de las instituciones que operan el recurso hídrico forman parte del sector público, pero debido a que se trata de muchos organismos abasteciendo de agua a la población, en distintos lugares, existen diversas formas de gestión del recurso. Esto ha tenido repercusiones negativas en la población, razón por la cual se confirma la necesidad de contar con líneas estratégicas sobre la administración, el aprovechamiento y la conservación del recurso que generen armonía en la manera de administrar el agua.

Dentro del escenario más amplio de actores que participan en la gestión del agua en Costa Rica, el caso particular de los acueductos municipales, aunque no se realizó un estudio histórico exhaustivo, sí permite notar una tendencia histórica donde este tipo de acueductos existe incluso previo a la fundación de cada cantón o jugó un papel importante en el desarrollo y crecimiento poblacional. Destacándose que la mayoría puede tener más de 50 años de funcionamiento.

Siendo los gobiernos locales actores políticos claves en el desarrollo del país, la investigación realizada estudia los cantones que cuentan con acueductos municipales. Cabe señalar que en algunos cantones se mantuvo acueductos por razones estratégicas o tradición, mientras que, en otros, dejaron de existir probablemente por falta de recursos, centralización del servicio en el AyA, problemas de gestión operativa y administrativas, entrada en escena de Asociaciones Administradoras de Acueductos, entre otras.

El estudio de estos acueductos municipales parte de una visión de gestión integral del recurso hídrico, ya que desde su conceptualización resulta ser una alternativa de adaptación al cambio climático con la que se espera maximizar el bienestar social y económico 
de manera equitativa y sin comprometer la sostenibilidad de los ecosistemas vitales. Aunque en el presente artículo se parte de una visión utilitaria y economicista del agua, se debe señalar que existen contradicciones y limitaciones en cuanto a las conceptualizaciones aquí presentadas, con lo cual, no se deben considerar como verdades absolutas y puede darse variación en lo que se pueda entender por gestión integral del recurso hídrico de acuerdo con el posicionamiento ideológico que se tenga al respecto.

En los siguientes dos apartados se presentan aspectos de la metodología aplicada en el proceso de investigación, así como elementos conceptuales y teóricos en los cuales se sustentó la investigación principal, y que son relevantes para enmarcar teóricamente el aprovechamiento del recurso hídrico.

\section{Metodología aplicada en el proceso de la investigación}

El trabajo de la investigación principal se realiza desde un tipo de investigación descriptiva, con un enfoque cualitativo, entre mediados del año 2016 y finales del 2018, teniendo como unidad de estudio todas las municipalidades que brindan el servicio de agua mediante su acueducto municipal. En específico, se trabaja con las unidades encargadas de cada municipalidad de brindar este servicio.

Se consideraron en el estudio veintinueve municipalidades que contaban con acueductos municipales en todo el territorio nacional, excepto en la provincia de Limón. No obstante, del proceso de consulta, se logra obtener información de las siguientes diecinueve municipalidades: Aserrí, Dota, León Cortés, Tarrazú, Naranjo, Poás, San Carlos, Sarchí, Zarcero, Alvarado, Cartago, Jiménez, La Unión, Oreamuno, Paraíso, Cervantes, Flores, Montes de Oro, y, Abangares.

Se parte de limitaciones de carácter administrativas, económicas y técnicas presentes en la gestión del recurso hídrico a nivel país, y se plantea la pregunta: ¿Cómo se realiza la gestión del recurso hídrico en los acueductos municipales del país en lo referente a la administración, el aprovechamiento y la conservación?

Tal pregunta surge con la intención de analizar los procesos sustantivos de gestión del recurso hídrico. Esto considerando el comportamiento de los elementos administrativos, de aprovechamien- 
to y conservación en la práctica existentes en cada cantón. Se toma como punto de partida el hecho que es indispensable comprender la dinámica de gestión del recurso desde los gobiernos locales, los cuales son el primer contacto de la administración pública con la ciudadanía.

Para cada eje se elabora un instrumento para la sistematización de la información, el cual fue aplicado utilizando la revisión bibliográfica o documental, $\mathrm{y}$, un análisis de contenido. Las fuentes documentales se obtuvieron por medio de solicitudes por correo electrónico o descarga desde el sitio web de cada municipalidad. Dentro de las fuentes documentales se encontraban: planes estratégicos, planes anuales operativos, informes de resultados, entre otros.

Posteriormente, se hizo un análisis y validación con entrevistas a profundidad que se realizaron desde el mes de junio hasta el mes de setiembre del año 2018 a las personas encargadas de los acueductos municipales. Algunas entrevistas se hicieron realizando visitas de campo, otras vía telefónica, y algunas mediante consultas por correo electrónico.

Al momento de la redacción de este artículo en el año 2020, se realiza un acercamiento a las municipalidades que demostraron resultados en el 2018 con relación específicamente al aprovechamiento del recurso hídrico, sin embargo, al cotejar la información, se mantienen los resultados encontrados en la investigación original, por ello se presentan aquí tales resultados.

\section{Elementos conceptuales y teóricos relevantes}

En este apartado se presenta brevemente los elementos conceptuales más relevantes para que la persona lectora comprenda el posicionamiento del discurso de quienes elaboraron el presente artículo en relación con la gestión integral del recurso hídrico, y, propiamente, al aprovechamiento del recurso hídrico.

Gestión integral del recurso hídrico y el aprovechamiento del recurso hídrico

Es necesario destacar que los elementos conceptuales contenidos en los preceptos de la Gestión Integral de los Recursos Hídricos 
(GIRH) y aprovechamiento del recurso hídrico son más amplios de los que aquí se destacan. La idea en este apartado es puntualizar la conceptualización que fue utilizada en la investigación principal y que se mantiene en el presente artículo. Se invita a las personas lectoras, de tener interés en profundizar en esta conceptualización a realizar revisión de los textos que se citan en este espacio.

En la búsqueda de un marco conceptual para darle un sustento vertebral al trabajo de investigación, se encuentra un trabajo realizado precisamente sobre la aplicación de la Gestión Integral de los Recursos Hídricos a nivel municipal realizado por Yamileth Astorga en el 2013. En ese trabajo, Astorga (2013) cita a Global Water Partnership, indicando que la Gestión Integral de los Recursos Hídricos (GIRH) es vista como "un proceso que promueve el manejo y desarrollo coordinado del agua, la tierra y los recursos relacionados, con el fin de maximizar el bienestar social y económico resultante de manera equitativa, sin comprometer la sustentabilidad de los ecosistemas vitales" (p. 16).

De forma complementaria, Astorga (2013) señala que el significado de GIRH es que "todos los usos del agua deben ser considerados de forma integrada para su administración, aprovechamiento, y, conservación, siendo la unidad lógica de gestión, la cuenca hidrográfica" (p. 16). De ahí se parte a cada eje trabajado en la investigación principal: administración, aprovechamiento, y, conservación.

Se puntualiza, para términos de este artículo, que de acuerdo con Astorga (2013), el aprovechamiento del recurso hídrico, se relaciona con el volumen de agua de entrada al sistema, o cantidad de lluvia que ingresa en la cuenca, menos el volumen de salida. En relación con esto, el MINAE (2012) también señala que, el aprovechamiento consiste en "que se mantenga un equilibrio entre la oferta y demanda, dentro de criterios que otorguen prioridad al uso del consumo humano" (p. 34).

Teniendo presente que el aprovechamiento del recurso hídrico es fundamental para que, en este caso, los acueductos municipales no solo estén alineados a modelos de gestión pública que permita que el aprovechamiento se dé de manera eficiente, eficaz, y, económica, sino que también se tenga un análisis enfocado a factores que componen el acceso a agua segura.

Sobre esto último, estos factores son necesarios al momento de analizar tanto la gestión integral del recurso hídrico, como el apro- 
vechamiento del recurso hídrico.

En la siguiente tabla se hace síntesis de los factores que componen el acceso a agua segura, posteriormente, se traen a discusión en el apartado de resultados de este artículo. Esta síntesis se hace apoyada en lo escrito por Madroñero (2006):

Tabla 1. Factores que componen el acceso a agua segura

\begin{tabular}{|c|c|c|c|c|}
\hline Cobertura & Cantidad & Calidad & Costo & $\begin{array}{l}\text { Cultura } \\
\text { Hídrica }\end{array}$ \\
\hline $\begin{array}{l}\text { Significa } \\
\text { que el agua } \\
\text { debe llegar } \\
\text { a todas las } \\
\text { personas } \\
\text { sin res- } \\
\text { tricciones. } \\
\text { Nadie debe } \\
\text { quedar } \\
\text { excluido } \\
\text { del acceso } \\
\text { al agua } \\
\text { de buena } \\
\text { calidad. }\end{array}$ & $\begin{array}{l}\text { Necesidad } \\
\text { de las perso- } \\
\text { nas de tener } \\
\text { acceso a una } \\
\text { dotación de } \\
\text { agua sufi- } \\
\text { ciente para } \\
\text { satisfacer sus } \\
\text { necesidades } \\
\text { básicas } \\
\text { (OPS, 2004). }\end{array}$ & $\begin{array}{l}\text { Se define por } \\
\text { su composi- } \\
\text { ción química } \\
\text { y por sus } \\
\text { característi- } \\
\text { cas físicas y } \\
\text { biológicas, } \\
\text { adquiridas a } \\
\text { través de los } \\
\text { diferentes pro- } \\
\text { cesos naturales } \\
\text { y antropogé- } \\
\text { nicos. }\end{array}$ & $\begin{array}{l}\text { Incluye el } \\
\text { tratamiento, } \\
\text { el manteni- } \\
\text { miento y la } \\
\text { reparación de } \\
\text { las instalacio- } \\
\text { nes, así como } \\
\text { los gastos } \\
\text { administra- } \\
\text { tivos que un } \\
\text { buen servicio } \\
\text { exige. }\end{array}$ & $\begin{array}{l}\text { Implica el } \\
\text { compromiso } \\
\text { de valorar } \\
\text { y preservar } \\
\text { el recurso, } \\
\text { utilizándolo } \\
\text { con respon- } \\
\text { sabilidad en } \\
\text { todas las acti- } \\
\text { vidades, bajo } \\
\text { un esquema } \\
\text { de desarrollo } \\
\text { sustentable } \\
\text { (OPS, 2004). }\end{array}$ \\
\hline
\end{tabular}

Fuente: Agüero, Aragón, Fernández (2018), basado en Madroñero 2006.

Estos factores se utilizaron en la estructura de la investigación principal con la finalidad de cotejar su cumplimiento en cada eje de trabajo, en este artículo solo se presentan los resultados del eje de aprovechamiento del recurso hídrico. Cada factor en sí mismo, representa acciones que los acueductos municipales deben realizar para garantizar el servicio a su ciudadanía.

En el apartado siguiente se presentan los resultados principales, exclusivamente sobre lo encontrado en relación con el aprovechamiento del recurso hídrico en las diecinueve municipalidades en las que se obtuvo la mayor cantidad de información.

\section{RESULTADOS PRINCIPALES RELACIONADOS AL APROVECHAMIENTO DEL RECURSO HÍDRICO EN LAS MUNICIPALIDADES INVESTIGADAS}

Este apartado se estructura en función de los factores sintetizados 
de Madroñero (2006) que componen el acceso a agua segura, mencionados anteriormente en el apartado de elementos conceptuales y teóricos relevantes. Seguido por los resultados más relevantes en relación con los modelos de gestión pública. En la presentación de resultados, para facilitar la comprensión de quienes leen este artículo, podrán identificar algunas referencias bibliográficas que pudieran consultar para más información.

Los resultados se presentan en función de la cobertura y cantidad, la calidad y el costo, y la cultura hídrica.

Antes de entrar en detalle, es importante mencionar que el aprovechamiento del recurso hídrico debe identificarse como el uso de los recursos existentes, para ser desarrollados y asignados, garantizando el acceso equitativamente para todos los sectores y usuarios, preservando su cantidad, calidad, continuidad y seguridad (Agüero, Aragón, y Fernández, 2018). En este caso el enfoque es en el aprovechamiento de agua potable para consumo humano.

Por lo que, para poder cumplirse este aprovechamiento, se deben considerar una serie de elementos dentro de la gestión de los acueductos, por ejemplo: la infraestructura hidráulica. En la gestión del recurso hídrico es necesario que se considere el uso de una infraestructura y tecnología capaces de disminuir el desperdicio y la contaminación del agua.

De tal manera, que esta infraestructura se debe entender, según se señala en el documento Programa de Agua (s.f.), como el conjunto de equipo e instalaciones que se requieren para gestionar el recurso hídrico, que garanticen su aprovechamiento sostenible, eficiente tanto para la prestación de servicios como para su conservación. De acuerdo con Sahagun (2013), esta infraestructura hidráulica incluye, entre otros: las represas y embalses; la infraestructura básica para el abastecimiento y saneamiento (presas, acueductos, tuberías, depósitos, sistemas de monitoreo de calidad); y, la infraestructura para el tratamiento de aguas residuales (alcantarillado, plantas de tratamiento, sistemas de reutilización).

De esta infraestructura hidráulica las municipalidades que cuentan con acueductos municipales quedan debiendo. En relación con represas y embalses, la realidad es que las municipalidades cuentan con el canon que les permite contar con el recurso hídrico para la distribución de este a la ciudadanía. Barahona (2010) menciona que el acueducto es un componente de toda la infraestructura hi- 
dráulica, por ello, se explica que un sistema de acueducto consiste en el conjunto de obras que recogen el agua desde las fuentes de aprovisionamiento, y las lleva por medio de tuberías a cada vivienda o a una fuente de uso público.

Este recurso las municipalidades lo reciben, en su mayoría, directamente del Instituto Costarricense de Acueductos y Alcantarillados. Por lo que sí cuentan con la infraestructura básica de acueductos, tuberías, depósitos, y, sistemas de monitoreo de calidad. También se identifican debilidades en lo relacionado a la infraestructura para el tratamiento de aguas residuales, un asunto que al país aún le falta por avanzar.

Relacionado a esta infraestructura hidráulica, en el documento de la Comisión Nacional de Agua (2014), se estipula como algo necesario, la posibilidad de realizar macro medición y micro medición del agua, dado que son procesos útiles tanto para la administración del servicio, como para mostrar y registrar la eficacia del recurso en términos de calidad y continuidad. Entonces, relacionados con los puntos que siguen a continuación, el tema de macro medición y micro medición están estrechamente conexos con la cobertura y la cantidad para el aprovechamiento del recurso hídrico.

\section{Cobertura y cantidad}

En materia de cobertura y cantidad se deben tomar en cuenta aspectos de macro medición y micro medición. La primera busca cuantificar y registrar los caudales y volúmenes de agua que se requieren para proveer el servicio, $y$, la segunda busca cuantificar el consumo del recurso hídrico por parte de la ciudadanía. Es importante resaltar que, en la mayoría de los acueductos municipales investigados, la cobertura del servicio de agua potable se concentra en el distrito central de cada cantón.

\section{Macro medición}

En línea con lo que explica la Comisión Nacional de Agua, del Gobierno de México (2014), la macro medición se define como el conjunto de elementos y actividades que se destinan a la obtención, desarrollo, análisis y divulgación de los flujos, volúmenes, presiones y niveles de sistemas de aprovisionamiento de agua potable. 
Los servicios se consumen por peso, tamaño o volumen, por lo cual es imprescindible para los acueductos llevar un control operacional de las fuentes de aprovisionamiento, en torno al caudal, la presión y el nivel (Comisión Nacional de Agua, 2014). De la misma manera, resulta imprescindible registrar las pérdidas de agua ${ }^{1}$, ya sea llevando control del volumen de consumo, así como de la presión.

Para la Comisión Nacional de Agua (2014), el objetivo fundamental de los sistemas de macro medición es:

cuantificar y registrar los caudales y volúmenes de agua que se captan, potabilizan, conducen, regulan, y distribuyen en los sistemas de abastecimiento, incluyendo la medición de presiones y niveles, con fines de administración de la producción y de control operacional de la infraestructura, así como de su planificación, diseño, construcción, y mantenimiento. (p.4)

Por ello, se hizo relevante en la investigación determinar si se contaba con un registro de pérdidas de agua. El resultado es que la mayoría de los acueductos municipales no llevan un registro de pérdidas de agua ${ }^{2}$.

En cuanto a la cantidad de acueductos municipales que llevan o no este registro, doce de ellos no cuentan con registro (Abangares, Alvarado, Aserrí, Jiménez, Montes de Oro, Oreamuno, Paraíso, Poás, San Marcos de Tarrazú, Santa María de Dota, y Valver-

1 Estas pérdidas de agua tienen múltiples causas, entre ellas, las fugas o el consumo no contabilizado. En el Instituto Costarricense de Acueductos y Alcantarillados existe el Programa Reducción de Agua no Contabilizada y Optimización de la Eficiencia Energética, el cual tiene como objetivo alcanzar una reducción consistente del agua no contabilizada, integrando acciones dentro de los procesos de desarrollo, operación, mantenimiento y comercialización; garantizando inversiones y recursos que aseguren la continuidad de las acciones a largo plazo (Instituto Costarricense de Acueductos y Alcantarillados, 2018). No obstante, se desconoce el alcance que este programa pudiera tener con los acueductos municipales sujetos del estudio, ya que las inversiones están destinadas para la red de acueductos del propio AyA.

2 En el caso de estos acueductos municipales que no contabilizan en el cien por ciento la pérdida del agua, se suman a los datos analizados en la noticia "Agua hay, pero ¿por qué nos falta?" elaborado por Monserrat Cordero Parra y Javier Córdoba para el Semanario Universidad el 17 de marzo de 2020, donde se señala que, al menos el AyA, no contabiliza el 53\% de lo que produce y se gasta por consumo no contabilizado o fugas. Con lo cual se vuelve un reto para el país que se logre contabilizar la pérdida de agua. 
de Vega), mientras que siete de ellos sí evidenciaron contar con este (Cartago, Cervantes, Flores, La Unión, Naranjo, San Carlos, y Zarcero). La falta de registro puede suponer que no es posible controlar de manera adecuada el estado del recurso hídrico en la infraestructura hidráulica de los acueductos.

Esto es preocupante en dos sentidos, por un lado, representa pérdidas materiales y financieras para los acueductos, $y$, por otra parte, significa desperdicio del recurso hídrico, ya que no es posible gestionar de manera adecuada lo que no se conoce.

No obstante, esto puede deberse a una falta de presupuesto para ejecutar este tipo de proyecto, o por falta de talento humano capacitado, así como complicaciones en la geografía del territorio.

\section{Micro medición}

La micro medición permite contar con información fundamental para los encargados de acueductos municipales, tales como el número de abonados al servicio y su consumo histórico. Además, la Comisión Nacional de Agua (2014), señala que la micro medición adecuada posibilita la generación de mayores ingresos por la medición y facturación del agua consumida, así como la aplicación de una tarifa justa de acuerdo con el volumen de agua consumida.

Para lo anterior, Pérez, Fuertes, López y Herrera (2006), plantean que las tarifas que se apliquen a servicios públicos consisten en determinar una estructura que maximice el bienestar social, la eficiencia económica de la asignación y autofinanciación, así como recuperar los costos que supone prestar el servicio, a la vez que se tiene en cuenta las restricciones económicas, financieras y sociales de la población.

Sin dejar de lado, como lo explica el Consejo Mundial del Agua (2000), cobrar lo necesario de modo que los usuarios valoren y se hagan responsables del uso que se le da al recurso. De ahí la importancia de prestar un servicio que garantice que las personas hagan un uso consciente del servicio, y que genere los ingresos suficientes para operar y mantener los sistemas de acueducto. Lo cual podría permitir que se invierta en infraestructura o se investigue en nuevas tecnologías, ya que, de otro modo se puede inducir a un círculo vicioso en el que los sistemas sufren deterioro y requieren más rehabilitación de lo normal. 
En general, la medición es necesaria no sólo para la planificación, administración y control de los procesos del prestador del servicio, sino también para mostrar y registrar la calidad con que éste se presta a los usuarios, en cuanto a cantidad, continuidad y presiones de suministro (Comisión Nacional de Agua, 2014).

Por lo tanto, la micro medición se torna indispensable para la gestión del acueducto en tanto permite conocer el histórico de consumo de los abonados, pero además refleja la generación de ingresos equitativos al volumen de agua que se consume, por medio del ajuste de tarifas.

A diferencia de la falta de registro de pérdidas de agua, en este punto, doce acueductos cuentan con sistemas de medidores (Abangares, Cartago, Jiménez, León Cortes, Montes de Oro, Oreamuno, Poás, San Carlos, San Marcos de Tarrazú, Santa María de Dota, Valverde Vega, y Zarcero) y otros siete no cuentan con dicho sistema de medición (Alvarado, Aserrí, Cervantes, Flores, La Unión, Naranjo, y Paraíso).

De la investigación realizada se acotó que dentro de las razones por la que no se cuenta con medidores, se debe a situaciones en la mayoría de los cantones donde las tomas se encuentran en terrenos privados a los que el acueducto no tiene acceso, o, porque al momento de realizar la investigación se encontraban en trámites, $\mathrm{o}$, porque en algunos acueductos municipales se encuentran instalados los medidores, pero no se utilizan.

\section{Calidad y costo}

En este apartado se hace oportuno señalar los resultados sobre tres aspectos fundamentales en relación con calidad y costo: la continuidad del servicio, la potabilidad del agua, y la cantidad de abonados. Teniendo en cuenta que la calidad y el costo estará en función de la gestión correcta del acueducto municipal y, que se parte de la premisa de que así es en cada uno de los casos estudiados.

\section{Continuidad del Servicio}


La continuidad del servicio se toma como un proceso que debe estar formalmente institucionalizado en las organizaciones operadoras del recurso para asegurar el suministro de agua potable a la población en caso de alguna emergencia o por efectos de mantenimiento en la infraestructura hidráulica. La continuidad del servicio se conoce como un

proceso de gestión holístico que identifica las amenazas potenciales de una organización y los impactos que pueden causar en las operaciones del negocio si esas amenazas se materializan. Además, proporciona un marco de trabajo para construir una organización más resistente con capacidad para responder de forma efectiva y proteger con los intereses de las partes interesadas. (Bereau Veritas, 2012, p.6)

En este punto, la mayoría de los acueductos no contaban con un plan establecido que instaurara con claridad los pasos a seguir para que se garantice la continuidad del servicio. Pareciera ser que en la mayoría se dan situaciones que los llevan a reaccionar de distintas maneras, incluyendo: buscar ayuda del Instituto Costarricense de Acueductos y Alcantarillados (AyA), buscar ayuda del Ministerio de Obras Públicas y Transporte, activar unidad de obras públicas municipal, recurrir a los acueductos comunales para poder continuar con servicio de manera temporal, entre otras.

\section{Potabilidad del agua}

Sobre este punto se consultaron los resultados del Informe de Calidad del Agua, emitido por el AyA, donde se expone que todos los acueductos muestreados superan los estándares planteados en el Reglamento para la Calidad del Agua Potable, y del mismo modo, se ha mantenido el nivel en la calidad del agua durante el periodo estudiado (2015-2016) (ICAyA, 2016). Por lo cual, se reconoce que la totalidad de acueductos entrevistados garantizan la prestación del servicio con una potabilidad buena o excelente.

\section{Cantidad de abonados}

Los resultados de la investigación reflejan que siete de los diecinueve acueductos municipales estudiados atienden entre 2.000 a 6.000 
abonados (Santa María de Dota, San Marcos de Tarrazú, Alvarado, Montes de Oro, Poás, Naranjo, y Aserrí), cinco acueductos atienden tanto menos de 2.000 (León Cortes, Zarcero, Jiménez, Cervantes, y Abangares), otros cinco atienden a más de 10.000 (San Carlos, Paraíso, Valverde Vega, La Unión, y Cartago), y solo dos atienden entre 6.000 a 10.000 abonados (Flores y Oreamuno).

Figura 1. Cantidad de acueductos municipales de acuerdo con la cantidad de abonados que atienden, 2018

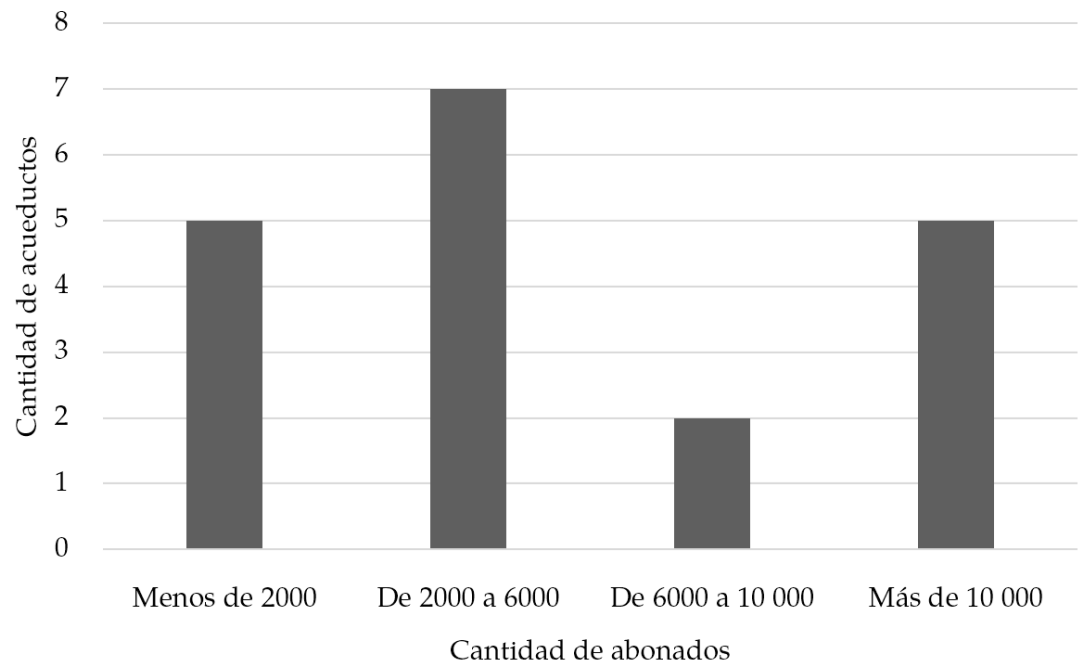

Fuente: Agüero, Aragón, Fernández (2018).

Esto comprueba que la cantidad de abonados es diversa, como se aprecia en la Figura 1. Además, esto se debe en parte al alcance territorial que se tiene en lo que son los distritos centrales de cada cantón.

De esta información también es posible indicar que, los funcionarios de los acueductos deben atender una cantidad de abonados que puede ser considerada desproporcional entre sí. Esto se debe al hecho de que cada funcionario puede estar atendiendo una cantidad mínima de 149 abonados o una máxima de 1067.

Esto puede ser causado por distintas razones, por ejemplo, por falta de recursos económicos o presupuestarios, automatización de procesos, por el tamaño del territorio, o por malas condiciones de 
la infraestructura hídrica. Así como también se puede relacionar con una dependencia debido al tamaño de la municipalidad, y si se encuentra en zona urbana o rural.

\section{Cultura hídrica}

La cultura hídrica o del agua se puede definir como el "conjunto de saberes, valores, costumbres, actitudes y hábitos construidos con las comunidades y demás actores para una conciencia social hacia el uso racional, justo, disponible y eficiente del agua potable" (ICAyA, 2016, p. 65).

Por lo cual, se considera de gran importancia el elemento de educación y empoderamiento sobre el uso y protección del agua, ya que educar a la población en estos temas les brinda herramientas para que sepan utilizar el recurso hídrico.

De acuerdo con Bedoy citado en (Olaguez, Peña y Espino, 2017), la educación ambiental implica un proceso integral y multidisciplinario que se conduce al entendimiento de la dinámica del ambiente aunado a la interacción de manera activa y organizada de las personas para ajustar sus modos de vida, brindando así las bases a una sociedad sustentable y socialmente justa.

En este apartado se presentan resultados que se pueden relacionar directamente con el aprovechamiento del recurso hídrico, estos son: fuentes de aprovisionamiento, reutilización de aguas, programas de vinculación con la comunidad, acciones para atender emergencias, y acciones para enfrentar el cambio climático.

\section{Fuentes de aprovisionamiento}

En relación con las fuentes de aprovisionamiento, la mayoría de los acueductos municipales estudiados obtienen el agua de nacientes (un total de 17), seguido por pozos (un total de 4), aguas superficiales (un total de 3), y otros (un total de 2).

Los siguientes acueductos municipales indicaron tener como fuente de aprovisionamiento únicamente las nacientes: Alvarado, Cervantes, Jiménez, León Cortes, Monte de Oro, Naranjo, Oreamuno, Paraíso, Poás, San Carlos, San Marcos de Tarrazú, Santa María de Dota, Valverde Vega, y Zarcero. Solo Flores indicó tener como fuente de aprovisionamiento únicamente los pozos. Otros acue- 
ductos municipales indicaron tener al menos dos fuentes de donde obtienen el recurso hídrico: Aserrí y Cartago obtienen el agua de nacientes, pozos y aguas superficiales; La Unión indicó obtener el agua de nacientes, pozos y otros; y, Abangares indicó obtener el agua de aguas superficiales y otros.

Resulta de especial interés que se obtenga el agua de las nacientes, pues este tipo de fuente es la más valiosa en tanto suele ser la menos contaminada, pero al mismo tiempo es vulnerable pues las malas prácticas humanas pueden tener un gran impacto en su deterioro e incluso agotamiento (Astorga, 2013). Pero, también se destaca que al ser fuentes de afloramiento natural muchas de ellas se encuentran actualmente en propiedad privada.

Junto a esto, respecto a si las fuentes son suficientes para satisfacer la demanda, la mayoría de los acueductos municipales (un total de 16) indican que sí (Abangares, Alvarado, Cartago, Jiménez, La Unión, León Cortes, Montes de Oro, Naranjo, Oreamuno, Paraíso, Poás, San Carlos, San Marcos de Tarrazú, Santa María de Dota, Valverde Vega, y Zarcero). Solo tres indicaron que no cuentan con la cantidad de fuentes suficientes para satisfacer la demanda (Aserrí, Cervantes, y Flores), esto se debe en parte por la ubicación geográfica de las fuentes en relación con la delimitación territorial del cantón. Esto quiere decir que hay lugares que deben recurrir a comprar terrenos en otros cantones, o dependen de las concesiones que se realicen para cada caso.

También es importante destacar que existe un esfuerzo de algunos acueductos por incorporar fuentes alternas a las nacientes dentro de su gestión, ya que este tipo de acciones garantizan seguridad hídrica en aspectos como la cantidad y la continuidad del recurso, aun cuando consideran que las fuentes de aprovisionamiento son suficientes para satisfacer la demanda.

\section{Reutilización de aguas}

En este punto, la investigación arrojó que la mayoría de las municipalidades no incluye dentro de su gestión la reutilización del recurso. Aun así, se pueden identificar cuatro municipalidades que tienen esta práctica en actividades como el riego y los hidrantes. De los resultados se destacan que un total de 15 acueductos municipales sí cuentan con iniciativas de reutilización de aguas (Alvarado, 
Cartago, Flores, Jiménez, León Cortes, Montes de Oro, Naranjo, Oreamuno, Paraíso, Poás, San Carlos, San Marcos de Tarrazú, Santa María de Dota, Valverde Vega, y Zarcero), mientras que un total de 4 indicaron que no contaban con iniciativas en esta materia (Abangares, Aserrí, Cervantes, y La Unión).

Es importante recalcar que estas acciones son necesarias para conseguir el máximo provecho del recurso, y se debe destinar capital para que esto pueda ser así, ya que se aprovecha mayoritariamente el agua potable para el consumo humano, pero también se podría aprovechar para otros usos el agua reutilizable.

\section{Programas de vinculación con la comunidad}

Sobre este punto, se identificaron once de los acueductos entrevistados que cuentan con proyectos de vinculación con la comunidad (Abangares, Alvarado, Cartago, Jiménez, La Unión, Montes de Oro, Paraíso, Poás, San Carlos, Valverde Vega, y Zarcero), por ejemplo: Bandera azul ecológica, campañas de sensibilización y uso racional, reforestación, rendición de cuentas de forma pública, presupuestos participativos, biojardinería y ferias ambientales.

Mientras que los otros ocho acueductos municipales no cuentan con programas de este tipo (Aserrí, Cervantes, Flores, León Cortes, Naranjo, Oreamuno, San Marcos de Tarrazú, y Santa María de Dota), esto puede representar una debilidad, tomando en cuenta que, para efectuar una mejora continua en la gestión, se requiere fortalecer la interacción de todos los actores involucrados en el tema.

Los programas de vinculación con la comunidad estimulan los mecanismos de gobernanza del recurso, por tanto, el involucramiento de distintos actores en la gestión del recurso promueve la eficiencia y eficacia de las tareas emprendidas. Por ello, a lo largo del tiempo ha adquirido un papel relevante en la gestión del acueducto. Un aspecto importante de mejorar.

\section{Acciones para atender emergencias}

En el proceso de la investigación se logró constatar que la mayoría de las acciones para atender emergencias son de carácter reactivas, 
es decir, satisfacen necesidades únicamente al corto plazo, como lo es el uso de cisternas (La Unión, San Marcos de Tarrazú, Abangares, Jiménez, Aserrí, Flores, Cartago, Alvarado, Paraíso, y Cervantes). En otros casos se cuenta con tanques de almacenamiento que entran en funcionamiento en el momento que surgen emergencias (León Cortes, Valverde Vega, y Santa María de Dota), el cual se encuentra en un proceso de aumento, donde se refleja que cada vez más acueductos invierten en su adquisición. En la siguiente figura se demuestra la cantidad de acueductos municipales que utilizan cada acción para atender emergencias.

Figura 2. Cantidad de acueductos municipales que utilizan cada acción para atender emergencias

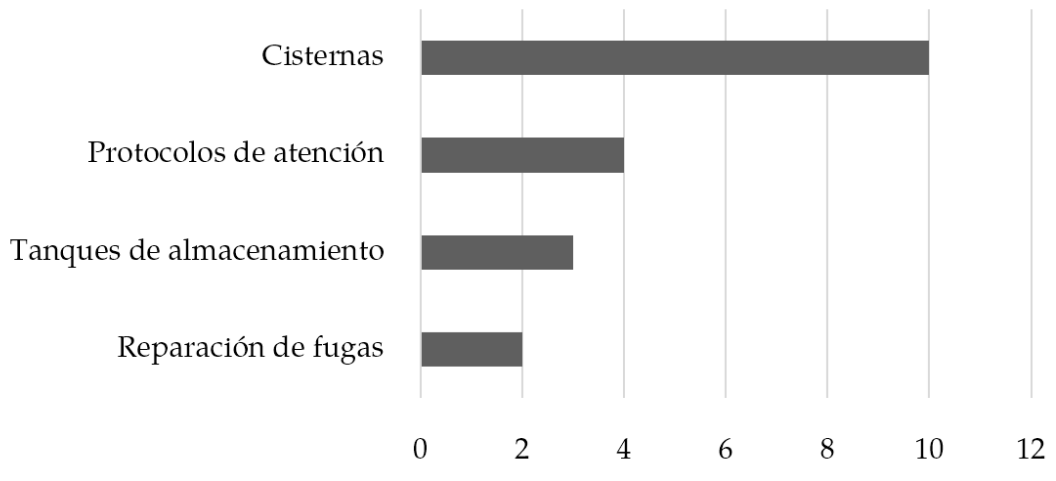

Fuente: Agüero, Aragón, Fernández (2018).

Por otra parte, también existen algunos protocolos de atención (Oreamuno, Poás, San Carlos, y Cartago) como la coordinación con otras municipalidades, el monitoreo constante de las tuberías y las interconexiones entre la infraestructura hídrica. Los anteriores representan un proceso de planificación previsor, en el cual se realizan acciones aún sin presentarse la emergencia de manera inmediata. San Carlos y Naranjo indicaron que las acciones para atender emergencias era la reparación se fugas.

Se logró evidenciar con la investigación que las acciones reactivas prevalecen sobre las preventivas. Por lo tanto, excluyendo a las comisiones municipales de emergencia, parece que en los propios acueductos municipales no hay una adecuada gestión del riesgo, 
pese a ello, se destaca que a nivel general las emergencias son reducidas y, en caso de que se presenten, sobresale el llevar a cabo acciones que se den de manera efectiva y eficiente para garantizar la continuidad del servicio, afectando en la menor medida posible a los usuarios del servicio.

\section{Acciones para enfrentar el Cambio Climático}

Sobre acciones para enfrentar el cambio climático, se identificó que existe una reducida planificación al mediano y largo plazo para contraatacar los posibles efectos de este. No obstante, se logró constatar que los programas de conservación del recurso y medidas alternativas para gestionarlo van en aumento.

Por lo tanto, a pesar de que ocho acueductos indicaron que no cuentan con acciones preventivas (Flores, León Cortes, Naranjo, Oreamuno, Paraíso, Valverde Vega, Zarcero, y Santa María de Dota), se reconoce el esfuerzo de los restantes municipios por invertir en proyectos de rearbolización y reforestación en zonas de vulnerabilidad ambiental (Abangares, Jiménez, San Carlos, Cartago, Montes de Oro, y La Unión). Además, se reconoce la inversión en infraestructura que permita mitigar la afectación a largo plazo, por medio de la construcción de tanques de almacenamiento y pozos, así como inversión en nuevas tuberías, es decir, medidas alternas para fortalecer la gestión del recurso (Alvarado, Aserrí, San Marcos de Tarrazú, La Unión, y Poás).

Esto permite considerar que, a través de los años, los modelos de aprovechamiento deben ser ajustados a la realidad medio ambiental del recurso, por lo que es necesario valorar elementos como estos desde la fase de planificación.

\section{CONSIDERACIONES FINALES}

El aprovechamiento del recurso hídrico se hace cada vez más importante. El cambio climático, la falta de contabilizar la pérdida del agua, la falta de planificación y de un modelo de gestión, al menos en los acueductos municipales estudiados, son algunas de las razones que pueden llegar a ser urgentes en el futuro. Si bien es cierto, los acueductos municipales son pocos entre diversos actores en Costa Rica involucrados en el aprovechamiento, administración 
y conservación, sí deben ser referentes y buscar siempre la calidad institucional al ser parte integral de los gobiernos locales.

No obstante, los acueductos municipales, sujetos a la investigación, permitieron identificar los elementos de la gestión del acueducto como lo es el aprovechamiento del recurso hídrico. Con relación a los 19 acueductos municipales alcanzados, se concluye que éstos han realizado importantes esfuerzos en esta materia y, que, aunque falta definir un modelo de gestión claro, tienen iniciativas interesantes para aprovechar el recurso hídrico, sin embargo, aún hay retos que deben superar.

Uno de los retos a atender está relacionado al cobro por tarifas, se determina que algunas de estas se cobran en conjunto con otros servicios municipales, lo cual distorsiona el valor real del servicio brindado y dificulta una gestión financiera adecuada. Junto con esto, se encuentra la presencia de medidores instalados sin funcionar o la inexistencia de estos se identifica como una debilidad que poseen los acueductos municipales, ya que la mayoría de sus ingresos son por esta vía y si no se cuenta con la identificación del consumo por abonado, no se estaría cobrando el servicio de manera correcta, $\mathrm{y}$, además, no permitiría un buen aprovechamiento del recurso. Esto, también implica que los usuarios no posean una cultura de ahorro del agua, ya que puede no haber conciencia en el valor del recurso.

En relación con el aprovechamiento, también es importante tener en cuenta las fuentes de aprovisionamiento, por lo estudiado, parece que existen inconformidades en la gestión de esta. La mayoría de las fuentes de aprovisionamiento son las nacientes, valiosas en cuanto a su calidad, sin embargo, son vulnerables. Aunque existen acciones para protegerlas de manera especial, también se puede encontrar que hay desconocimiento sobre las fugas en la infraestructura, ausencia de mecanismos que reconozcan un uso adecuado del recurso, falencias en el cobro, ausencia de mecanismos para reutilizar el agua, así como acciones para atender emergencias más reactivas que de prevención.

En cuanto a los ingresos para los acueductos municipales, en su mayoría provienen de las tarifas que se cobran a los usuarios por el servicio, sin embargo, se encontró que en algunas municipalidades las tarifas se encuentran desactualizadas o en el peor de los casos se cobra una tarifa fija sin tomar en cuenta el consumo por abonado. 
Por lo tanto, se vuelve una prioridad la instalación de medidores a la totalidad de los abonados y actualizar las tarifas como corresponda para mejorar la recaudación y contar con mayor dinero disponible para proyectos de inversión en el sistema de acueducto.

De igual manera, los ingresos por tarifas deben ser identificables y trazables. Su cobro se debe de realizar únicamente por ese concepto, ya que en algunos casos se cobra junto con el servicio de recolección de residuos e impuestos municipales, entre otros. Se debe contar con un sistema de cobro que separe por cuentas lo que corresponde al acueducto, ya que puede existir el riesgo de desfinanciamiento de este, si estos recursos se utilizan para otros asuntos.

Por ello, para poder garantizar un uso justo del recurso para y por todos los abonados, así como para poder tener conocimiento de la efectividad de las tarifas, se debe poder contar con medidas de mantenimiento preventivo de los medidores, que vayan más allá de inspecciones producto de reportes de averías, sino que se concentren en garantizar su buen funcionamiento diario, y no permitan ser herramientas útiles para medir el aprovechamiento de este recurso.

Para finalizar, es importante que en Costa Rica se trabajen en una conceptualización de la gestión integral del recurso hídrico, tomando en cuenta las particularidades del ecosistema de actores que existen. Si bien es cierto, el aprovechamiento es una parte medular, no se puede olvidar que la administración y la conservación del recurso hídrico son también igual de importantes. La investigación que sirvió de base para este artículo es una iniciativa para poder llegar a entender la integralidad en la gestión del recurso.

Por lo cual, se recomienda a la comunidad científica investigar sobre la prestación del servicio que incluya la percepción de los usuarios de los acueductos, así al integrar esta contraparte se visualizaría completamente el servicio público municipal de recurso hídrico. Del mismo modo se puede realizar un estudio a profundidad de cada acueducto, para conocer más detalles de cada uno, ya sea de forma individual, por provincia, o de otra forma que se considere pertinente. También se pueden realizar investigaciones en la misma línea metodológica enfocadas hacia la gestión de los acueductos rurales (ASADAS) y otros proveedores del servicio (AyA y ESPH). El aprovechamiento del recurso hídrico puede marcar la 
diferencia en el futuro, para Costa Rica es necesario tomarlo con seriedad desde ya, y los acueductos municipales bien preparados pueden ser referente para otras organizaciones en esta materia.

\section{REFERENCIAS BIBLIOGRÁFICAS}

Agüero Hurtado, S., Aragón Hernández, D. y, Fernández Ramírez, M. L. (2018). Análisis de la gestión local del recurso hídrico en los acueductos municipales: el caso de Costa Rica. [tesis de licenciatura, Universidad de Costa Rica]. Repositorio del SIBDI-UCR: http://repositorio.sibdi.ucr. ac.cr:8080/jspui/bitstream/123456789/7269/1/43656.pdf

Alguacil, J. (2012). Administración relacional. Intervención Psicosocial, (13), 289-305. http://www.seeci.net/revista/index.php/seeci/article/ view/ 9/pdf_1

Araya, E. y Cerpa, A. (2009). La nueva gestión pública y las reformas en la Administración Pública Chilena. Tékhne - Revista de Estudios Politécnicos, (11), 19-47. http://www.scielo.mec.pt/scielo.php?script=sci_arttext\&pid=S1645-99112009000 100003\&lng $=$ pt\&tlng=es.

Asamblea Legislativa de Costa Rica. (1995). Ley No. 7554. Ley Orgánica del Ambiente. https://www.pgrweb.go.cr/scij/Busqueda/Normativa/ Normas/nrm_texto_completo.aspx?param $1=$ NRTC\&nValor1=1\&nValor2 $=27738 \&$ nValor3 $=93505 \&$ strTipM $=\mathrm{TC}$

Astorga, Y. (2013). Guía para la aplicación de la Gestión Integrada del Recurso Hídrico (GIRH) a nivel municipal. http://www.gwp.org/globalassets/ global/gwp-cam_files/guia-girh-a-escala municipal.pdf

Barahona, A, (2010). Operación y mantenimiento de sistemas de abastecimiento de agua. https://cmsdata.iucn.org/downloads/3_5_fasciculo_4_ operacion_y_mantenimiento.pdf

Bereau Veritas. (setiembre 2012). ISO 22301 - Continuidad del Negocio. Mantenga funcionado su negocio sin importar qué pueda ocurrir. https://www. interempresas.net/FeriaVirtual/Catalogos_y_documentos/87942/ Continuidad_Negocio-ISO-22301.pdf

Carvajal-Escobar, Y., García-González, M. y Jiménez-Escobar, H. (2007). La gestión integrada de los recursos hídricos como estrategia de adaptación al cambio climático. Ingeniería y Competitividad, 9 (1), 19-29.

Campillo, C. (2012). La Administración Municipal Relacional y Participativa. Cómo Construir la Identidad de las Ciudades desde una Perspectiva de Comunicación Neopública. https://e-revistas.uc3m.es/index.php/ EU- 
NOM/article/ viewFile/2079/ 1012

Cejudo, G. (2011). Nueva gestión pública. 1era ed. Ciudad de México: Grupo editorial siglo veintiuno.

Chiavenato, I. (2004). Introducción a la teoría general de la administración, $7 m a$ Ed. México: McGraw-Hill.

Comisión Nacional de Agua (2014). Manual de Agua Potable, Alcantarillado y Saneamiento Sistemas de Medición del Agua: Producción, Operación y consumo. Tlaplan, México, D.F. http://cmx.org.mx/wp-content/ uploads/MAPAS\%202015/libros/SGAPDS-1-15-Libro9.pdf

Consejo Mundial del Agua. (2000). The Use of Water Today. http://www. worldwatercouncil.org/fileadmin/wwc/Library/WWVision/TableOfContents.pdf

Cordero-Parra, M. y Córdoba, J. (17 de marzo de 2020). Agua hay, pero ¿por qué nos falta? Semanario Universidad. https://semanariouniversidad.com/ultima-hora/agua-hay-pero-por-que-nos-falta/

Costa Rica. (1949). Constitución de la República de Costa Rica: texto oficial. Ed. bilingüe. San José, Costa Rica.

Echeverría, J. y Cantillo, B. (2013). Instrumentos económicos para la gestión del agua. Ambientales, (45). https://doi.org/10.15359/rca.45-1.2

González, A. (21 de junio de 2013). Municipios buscan afinar estrategia para mejorar sistemas de acueductos. La Nación. https: / /www.nacion. com/el-pais/servicios/municipios-buscan-afinar-estrategia-para-mejorar-sistemas-de-acueductos/ZJZAB45JZZELLOQMZSPBBSOBLI/ story/

Global Water Parthership Centroamérica (2003). Régimen del recurso hídrico. El caso de Costa Rica. https://www.gwp.org/globalassets/global/ gwp-cam_files/regimen-del-recurso-hidrico.pdf

Global Water Parthership. (2012). "Agenda del Agua" Costa Rica 2013 - 2030. http://www.gwp.org/Global/GWPCAm_Files/Documento_de_Posicionamiento_Agenda_del_Agua_nov_2012\%5B1\%5D.pdf

Guerrero, O. (2001). Nuevos Modelos de Gestión Pública. Revista Digital Universitaria, (2). http://www.revista.unam.mx/vol.2/num3/art3/index.html

Instituto Costarricense de Acueductos y Alcantarillados. (2016). Agua para consumo humano y saneamiento y su relación con los indicadores básicos de salud en costa rica: objetivos de desarrollo del milenio y la agenda para el 
2030. https:/ / www.aya.go.cr/centroDocumetacion/catalogoGeneral/ Informe\%20de\%20calidad\%20del\%20agua\%202015.pdf

Instituto Costarricense de Acueductos y Alcantarillados. (2018). Informe de Gestión 2014-2018 de la Presidenta Ejecutiva del AyA. San José, Costa Rica. https://www.aya.go.cr/Noticias/Documents/Informe\%20 final $\% 20 \mathrm{de} \% 20 \mathrm{Gesti} \% \mathrm{C} 3 \%$ B3n $\% 20$ AyA $\% 202014 \% 202018$.pdf

Kliksberg, B. (1999). ¿Cómo Modernizar el Estado y Formar los Gerentes Sociales Necesarios? Algunas Sugerencias Para La Acción. ¿DE BURÓCRATAS A GERENTES?, 1era Ed. Washington: Banco Interamericano de Desarrollo.

Loaiza, K. (2015). Análisis del cambio de la eficiencia y la productividad a través del tiempo de los acueductos municipales de Costa Rica [tesis de licenciatura, Universidad de Costa Rica]. Repositorio del SIBDI-UCR: $\quad$ http://repositorio.sibdi.ucr.ac.cr:8080/jspui/bitstream/123456789/10146/1/39513.pdf

Madroñero S. (2006). Manejo del recurso hídrico y estrategias para su gestión integral en la microcuenca Mijitayo, Pasto Colombia. [tesis de postgrado, Centro Agronómico Tropical de Investigación y Enseñanza]. http:// www.sidalc.net/repdoc/A0690e/A0690e.pdf

Mariñez, F. (2016). La dimensión relacional del gobierno abierto y el liderazgo colaborativo. Espiral, Estudios sobre Estado y Sociedad, 23, 47-87. http://www.scielo.org.mx/pdf/espiral/v23n65/v23n65a2.pdf

Ministerio de Ambiente y Energía. (2004). Estrategia para la Gestión Integrada de Recursos Hídricos en Costa Rica (EGIRH). http:/ /idbdocs.iadb. org/wsdocs/getdocument.aspx?docnum $=958172$

Ministerio de Ambiente y Energía. (2008). Plan Nacional de Gestión Integrada de Recursos Hídricos, (CR-T1010) (ATN/WP-9338-CR). http:// www.iadb.org/es/proyectos/project-information-page,1303.html?i$\mathrm{d}=\mathrm{cr}-\mathrm{t} 1010$

Morales, D. (2010). Recursos Hídricos - Costa Rica 2010. Centro del agua para América y el Caribe.

Morales, M., García, C. y Carreón, J. (2014). Hacia una Administración Consensuada de los Recursos Hídricos en Ecociudades. Interdisciplinaria, (31), 163-174. http://www.redalyc.org/articulo.oa?id=18031545010

Municipalidad de La Unión. (2018). Política Cantonal del Recurso Hídrico. Cartago, Costa Rica.

Núñez, S. (24 de noviembre de 2011). El agua como bien económico. ALAI. https://www.alainet.org/es/active/51107 
Olaguez, J., Peña, E. y Espino, P. (2017). La Gestión de la Educación Ambiental en las Organizaciones desde la Perspectiva de los estudiantes de la universidad politécnica del Valle del Évora, México. Holos, (8), 145-159. https://www.redalyc.org/pdf/4815/481554853012.pdf

Pérez, R., Fuertes, V., López, G. y Herrera, M. (2006, 5-7 junio). Establecimiento de tarifas del servicio de agua potable bajo un enfoque de sostenibilidad económica. Seminario Iberoamericano sobre Sistemas de Abastecimiento Urbano de Agua. Brasil. http://www.lenhs.ct.ufpb.br/html/ downloads/serea/6serea/TRABALHOS/trabalhoE.pdf

Petrella, C. (julio de 2007). Análisis de la Teoría Burocrática. Revista Electrónica de la Facultad de Ciencias Empresariales de la Universidad Católica del Uruguay. https://www.fing.edu.uy/catedras/disi/ DISI/pdf/Analisisdelateoriaburocratica.pdf

Prats i Català, J. (2005). La gobernanza como modo de gobernación de nuestro tiempo, $1^{a}$. Ed. España: INAP.

Programa de Agua. (s.f.). Infraestructura hidráulica*. Agua.org.mx. https://agua.org.mx/categoria/infraestructura-hidraulica/

Ramos, M. y León, A. (2016). Gestión integral de pérdidas de agua: un caso de estudio. Ingeniería Hidráulica y Ambiental, (37), 74-88. http://scielo. sld.cu/scielo.php?script=sci_arttext\&pid=S1680-03382016000300006

Ruano, J. (octubre de 2002). La gobernanza como forma de acción pública y como concepto analítico VII Congreso Internacional del CLAD sobre la Reforma del Estado y de la Administración Pública. Lisboa. http:// biblioteca.senplades.gob.ec/jspui/handle/30000/255

Sahagun, A. (2013). Infraestructura hidráulica. https://prezi.com/pttp70ftzoiv/infraestructura-hidraulica/

Ureña L. (2016). Evaluación de la oferta y demanda del recurso hídrico - propuesta de mejoras para el sistema de acueducto de San Isidro de El Guarco, Cartago, Costa Rica. [tesis de licenciatura, Instituto Tecnológico Costarricense, Cartago.]. Repositorio TEC: https://repositoriotec.tec.ac.cr/ handle/2238/6455

ORLANDO JOSUÉ HERNÁNDEZ CRUZ es costarricense. Doctor en Gobierno y Políticas Públicas por la Universidad de Costa Rica. Director del Centro de Investigación y Capacitación en Administración Pública de la Universidad de Costa Rica. Profesor adjunto de la Escuela de Administración Pública de la misma universidad. 
MARIE LAURA FERNÁNDEZ RAMÍREZ es costarricense. Licenciada en Administración Pública por la Universidad de Costa Rica. Consulta independiente. Fue asistente del Programa de Desarrollo Municipal del Centro de Investigación y Capacitación en Administración Pública de la Universidad de Costa Rica. 